\title{
OPTICAL SEARCH OF FAINT IRAS POINT SOURCES IN THE VIRGO CLUSTER REGION
}

\author{
X. T. He and K. Liang \\ Department of Astronomy \\ Beijing Normal University \\ Beijing 100875, China
}

\author{
K. Huang \\ Institute for Astrophysics \\ Nanjing University \\ Nanjing 210008, China
}

\section{INTRODUCTION}

The infrared emission in galaxies is strongly connected to star burst processes, while emission from the intergalactic medium results mainly from dust heated by other luminosity sources. The Virgo cluster provides an extensive region in which the properties of such infrared emission can be studied. Galaxies in the Virgo cluster with dust temperatures of $40 \mathrm{~K}$ and masses of dust as small as about $5 \times 10^{4} M_{\odot}$ are detectable at the limit of the IRAS survey (Soifer et al., 1987). The IRAS observations of 196 optically selected galaxies in the Virgo cluster have been studied by Helou et al. (Helou et al., 1988; see also Leggett et al., 1988).

This paper presents a deep optical search for counterparts of faint IRAS point sources in the Virgo cluster region. The search area is about $100 \mathrm{deg}^{2}$. Three hundred fifty-seven infrared point sources are found from the data of the IRAS faint sources survey. The sources are identified using the UK Schmidt objective-prism and direct plates.

\section{THE IRAS DATA}

The IRAS Faint Source Survey (FSS) data have achieved a 2.5 -fold gain in sensitivity with respect to the Point Source Catalog by coadding the original data scans (Moshir et al. 1989). The main product of the IRAS FSS is the Faint Source Catalogue (FSC). The first release of the FSC contains 27,827 sources and is restricted to galactic latitudes $>50^{\circ}$. The Virgo cluster falls within this range. Three hundred fifty-seven sources are found from the FSC in about $100 \mathrm{deg}^{2}$ of the Virgo cluster region. For comparison, from the Point Source Catalog, only 206 sources are found in $113 \mathrm{deg}^{2}$ of the same region (Leggett et al. 1988).

Another product of the FSS is called the Faint Reject File (Moshir et al. 1989). The word "Reject" here emphasizes that the reliability of this database is significantly lower than that of the FSC. We have not worked with these data.

\section{THE IDENTIFICATIONS}

The objective prism plates and direct plates centered at $12^{\mathrm{h}} 25^{\mathrm{m}}$ and $13^{\circ} 30^{\prime}$ were taken by the UK Schmidt telescope in Australia. The region is divided into four areas. The identifications were carried out in the Kitt Peak Observatory with the PDS. One area has been searched so far, and 107 sources were detected. The identifications are shown in Table 1. 
Table 1. Identifications of One Area

\begin{tabular}{|c|c|c|c|c|c|}
\hline Number & Classification & $\begin{array}{l}\text { Identified } \\
\text { (Edinburgh) }\end{array}$ & New Iden. & $\begin{array}{c}\% \text { of } \\
\text { population }\end{array}$ & Remarks \\
\hline 31 & S. galaxies & 30 & 1 & 29.8 & \\
\hline 4 & E. galaxies & 4 & & 3.8 & \\
\hline 36 & galaxies & 5 & 31 & 34.0 & $\begin{array}{l}\text { galaxies not } \\
\text { classified }\end{array}$ \\
\hline 30 & $\begin{array}{l}\text { stars } \\
\text { (K:17, M:6, G:2, A:5) }\end{array}$ & 15 & 15 & 28.0 & types bracketed \\
\hline 5 & UVX objects & & 5 & 4.7 & $\begin{array}{l}\text { two are QSO } \\
\text { candidates }\end{array}$ \\
\hline 1 & no identification & & & & \\
\hline
\end{tabular}

All of the sources except one have been identified from the objective prism plates. The rms positional precisions of sources in any case are less than $20^{\prime \prime}$. Our search box is about $10^{\prime \prime} \times 10^{\prime \prime}$. There are five sources associated with two objects. Seventy-one sources are identified as galaxies, which is $66.4 \%$ of the total population. Thirty-five are stars, which is $32.7 \%$ of the total population.

\section{DISCUSSION}

The optical search shows that all the IRAS faint sources may potentially be identified from the UK Schmidt objective prism plates. The plates have a magnitude limit of $B=21$. It is not difficult to classify the optical objects even from a very low resolution spectrum. Therefore, this survey provides an efficient technique that can be of substantial use in the identification of the IRAS faint sources.

For bright galaxies, the IRAS galaxy sources are dominated by spirals. The galaxies with magnitude B > 16 are mostly blue compact dwarf galaxies. The faintest galaxy detected is about 20.5. The infrared emission from those optical faint galaxies indicates that they are undergoing a period of active star formation. Indeed, we have observed a number of these with the MMT. More that $60 \%$ show strong emission lines.

The fraction of stars in our survey is about $33 \%$, which is significantly increased from previous estimates. The fraction of stars for the Point Source Catalog is only about $27 \%$ (Leggett et al. 1988).

Acknowledgments-We thank Professor Tom Soifer for hospitality during our stay at IPAC and acknowledge help from Drs. W. Rice, J. Good, and L. Fullmer. The optical identifications were carried out at the Kitt Peak National Observatory. This project was supported in part by NSF grant INT-8600806 and the National Natural Science Foundation of China.

\section{REFERENCES}

Helou, G. et al. 1988, Ap. J. Suppl., 68, 151.

Moshir, M. et al., ed. 1989, IRAS Faint Source Survey.

Leggett, S. K. et al. 1988, MNRAS, 227, 563.

Soifer, B. T., Houck, J. R., and Neugebauer, G. 1987, Ann. Rev. of Astron. \& Astrophys., 187. 